\title{
Regulate the Efficiency of Cardiac Pacemaker based on Predictive Controller and Neural Predictive Controller
}

\author{
Noor Safaa Abdul-Jaleel \\ Assistant Lecturer \\ Department of Electrical Engineering \\ Al-Mustansiriayah University
}

\begin{abstract}
Heart disease is one of more serious diseases which are considered as the first reason that causes deaths in the world. Therefore, the need arises to find new ways to maintain the safety of patients. The pacemaker is the important device that helps for regulating the heart rate and ensures its survival in the normal range of human heart. This paper proposed methods to control the pacemaker based on the model predictive control and neural predictive control. The results show the model predictive control with neural network gives the better performance with zero overshoot.
\end{abstract}

\section{Keywords}

Model predictive control, neural predictive control, pacemaker, heart rate.

\section{INTRODUCTION}

Heart disease considered as the most dangerous diseases that may infect human and the primary reason for many of the deaths in the world. Early detection of heart disease and appropriate treatment considered as main factors of contribute to reduce the incidence of sudden death of the patient. So there was a need to find new ways to reduce the risk of this disease. Many of the modern methods and theories are adopted to reduce its severity and save the patient as soon as possible. A pacemaker is a medical device used to regulate heartbeat in case of any defect in the natural functions of the heart [1]. The pacemaker consists of two basic units: "sensing circuit" for sensing the heart rate of the patient and the "output circuit" for sending generated signals to the muscles of the heart [1]. The pacemaker monitors the heart rate and sends small electrical signals and is painful to the right ventricle of the heart to start each stroke of the heart only when needed. So, the main purpose of the pacemaker is to maintain the heart rate enough, either because the speed of normal heart pacemaker is insufficient, or that the electrical conductivity of the heart is Unemployed. Pacemaker modern programmable allow the cardiologist to identify specific formula for each patient individually. Some devices contain a pacemaker and defibrillator in a single device. Others contain multiple poles serve to alert the disparate locations within the heart and in turn improve the synchronization of the ventricles of the heart. The (ECG) Electrocardiography is a recurring sequence consisting of $(\mathrm{P}, \mathrm{T})$ waves and $(\mathrm{QRS})$ which is represent the three waves (Q, R and S) [2], as in Figure 1.

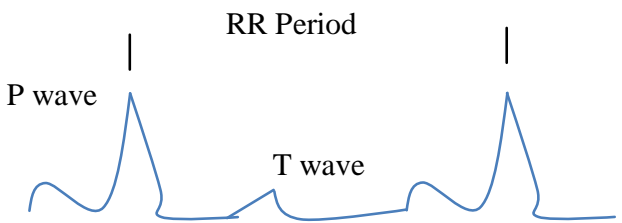

Fig. 1: QRS complex [1]
Where, RR is the period of ECG. A lot of measures have been taken to help patients recover and prevent the deterioration of the health situation. The most important of these measures, especially for intensive care patients monitor changes that might be exposed the heart by a pacemaker to inform physician of the need to take the necessary measures to save the patient's life. Many research are suggestion the methods for development the controller of the cardiac pacemaker to make the heart work normally. [3] Using the method of neural-fuzzy to control the rate of heart, which is found that this method is accurate more than the traditional method of fuzzy look-up table. [4] The authors design efficient method for controlling and using the pacemaker to regulate the HR. FLC was developed in [5] for controlling the heart rate of the pacemaker. Other algorithms for controlling the pacemaker are presented in [6-7].

\section{THE MODEL OF THE SYSTEM}

The closed loop of the cardiac pacemaker for controlling the rate of the heart is shown in Figure 2:

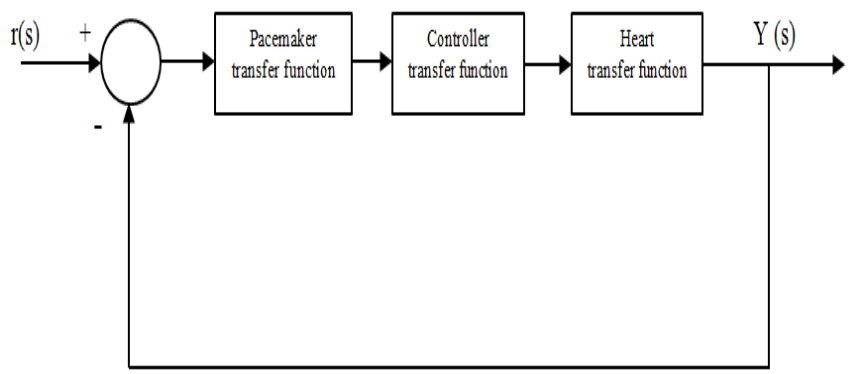

Fig. 2: The closed loop of the cardiac pacemaker for controlling the rate of the heart

The pacemaker's transfer function can be named as $G_{p}(s)$, $G_{c}(s)$ for the controller's transfer function, $G_{h}(s)$ represent the transfer function of the heart, $\mathrm{r}(\mathrm{s})$ the actual heart rate and $\mathrm{Y}(\mathrm{s})$ represent the output of heart rate.

The transfer function of the heart can be written as a system of second order from the mathematical model proposed in [7]:

$$
G_{h}(s)=\frac{169}{s^{2}+20.8 s}
$$

And the transfer function of the pacemaker can be written as a system of first order from the mathematical model proposed in [7]:

$G_{p}(s)=\frac{8}{s^{2}+8 s}$

Figure 3 show the closed loop of the cardiac pacemaker with the values of transfer functions 


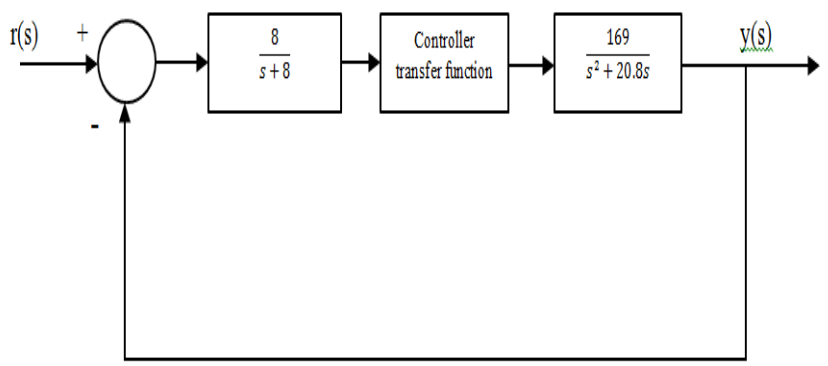

Fig. 3: The closed loop of the cardiac pacemaker for controlling the rate of the heart with the values of transfer function

The paper proposed model predictive control and neural predicative control for controlling the pacemaker to regulate the rate of heart as the next sections.

\section{MODEL PREDICTIVE CONTROL (MPC)}

MPC is a method of control and ways to adapt the systems. The property of this method is to enter future changes to the entrances of goals (ie. the value that we want to exit the system) in the calculation of exits uncontrolled change which makes this method is used in matters for tracing. The description of this method can be follow as:

1. Choose function or the path that will be following.

2. Calculated or solve control problem ideal any carry out to improve it, usually based on the sign of integration contains certain characteristics as a director controlled, where no need to the great effort to control the system. The integration typically contains differential or error between the track and the real track. In this area is minimized significance. .

3. Forward-looking way controller that works on digital computers later take the value obtained in the process above and used in step time pro. Although they can take this result for a longer period or the field to predict, but they do not take this step in the lead up to the time the pro at each step of the algorithm re-new. Therefore, checks the accounts are in the light of new data or real-time value for any new sensors.

The principle of control is the try to lower the value of the standard arithmetic or functional in each run of the model. This standard of the equation is discrete formula using a number of future $\left(n_{u}\right)$ entrances control, where: $\left(n_{l}\right)$ to reflect the minimum prediction horizon and $\left(n_{2}\right)$ for the maximum prediction horizon. The $(\rho)$ are standard balance to control the value of the second part of the equation on the amount of standard arithmetic total.

$u(k+1)=u(k+n), j=n+1, n+2, \ldots . r$

Where $n$ is the control horizon. Systems Nonlinear must calculate the optimization of the equation when each sample, leading to the prediction of a set of future values to the entrance of uncontrolled, of this group is entered the first value in the formula related to the first part of the function and the length of the segment is the length of time between the two improved to the system. As for linear systems is optimization in advance and transferred to the control predictor.

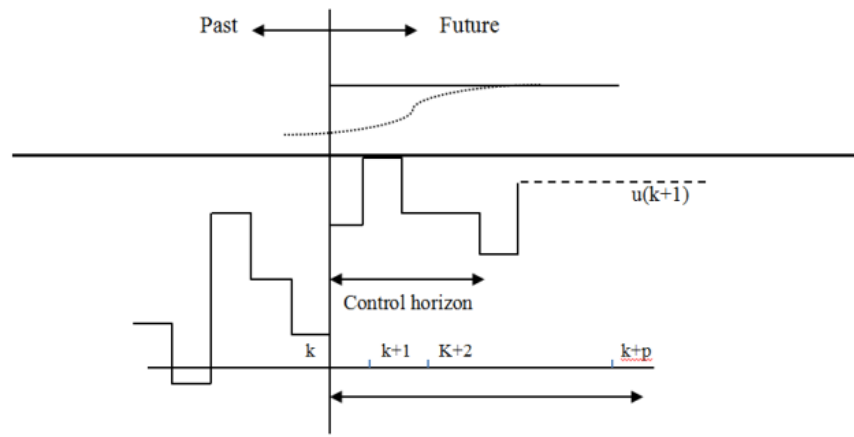

Fig. 4: The description of MPC [8]

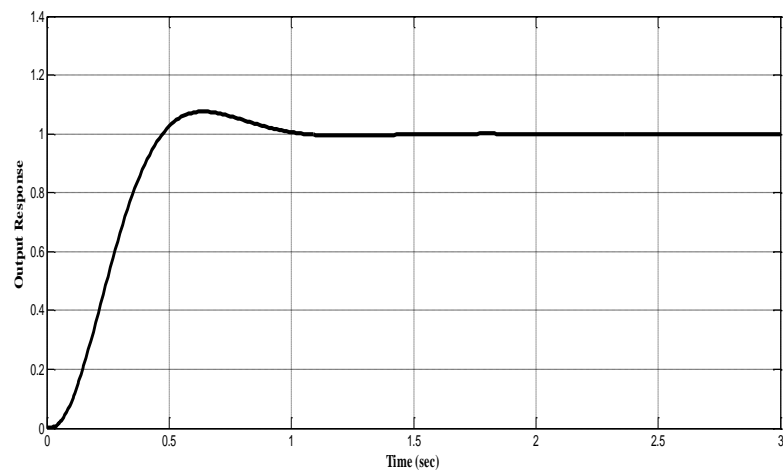

Fig. 5: The step response of the system with MPC

Figure 5 shows the output response of the system has overshoot. So, the neural network are using with the predictive control to get more accuracy and good result.

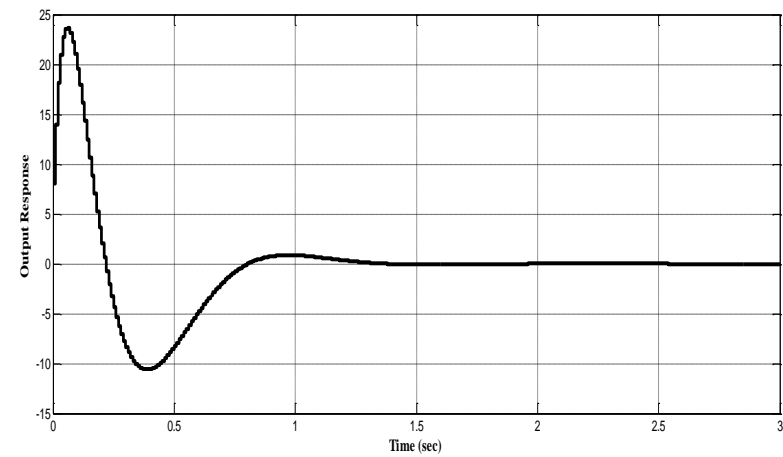

Fig. 6: The control signal

\section{NEURAL NETWORK MODEL PREDICTIVE CONTROL (NNMPC)}

By taking another case [9] the processes between the plant of the neural network and the output system are compared to produce the error signal for training the neural model [10]. The form of the model can be written as [10]:

$$
\begin{array}{r}
x_{k}(b+d)=h\left[x _ { k } \left((b), x_{k}(b-1), \ldots, x_{k}(b-n+1),\right.\right. \\
y_{c}(k), y_{c}(k-1), \ldots, y_{c}(k-m+1)
\end{array}
$$

$y_{c}$ : the input of the system, $x_{k}$ : the output of the system and $d$ : the delay of the system. 
First step is to find the output function $(y)$ by training the neural network to get the approximate function of y [11]. Then the neural training signal generated by prediction the error between the desired output $\left(x_{k}\right)$ and the output of the neural.

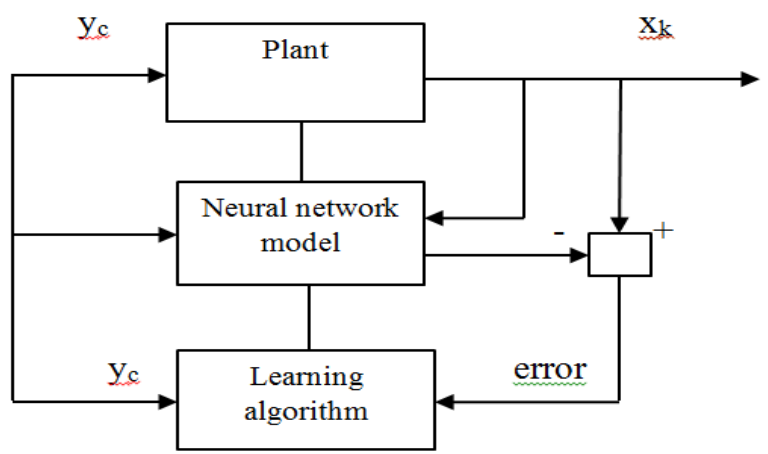

Fig. 7: The structure of model predictive neural control

Equation (5) represent the minimum cost function which is used the algorithm of the predictive optimization to produce the input signal [10]:

$$
\begin{aligned}
J= & \sum_{i=n 1}^{n 2}\left(x_{k r}(p+i)-x_{k m}(p+i)\right)^{2} \\
& +\left(y \sum_{i=1}^{n 0}\left(y_{c 0}(p+i-1)-y_{c 0}(p+i-2)\right)^{2}\right.
\end{aligned}
$$

Where: $n 1, n 2$ and $n 0$ is the horizons of the predictive error, $x_{k r}$ the output response and $x_{k m}$ is the response of the neural. Figure 8 shows the output response with zero overshoot and faster setting time compared to the system with MPC.

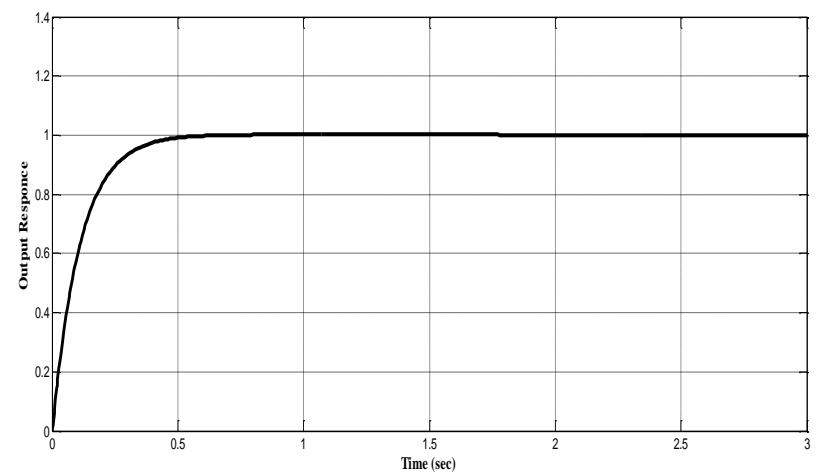

Fig. 8: The step response of the system with MPC

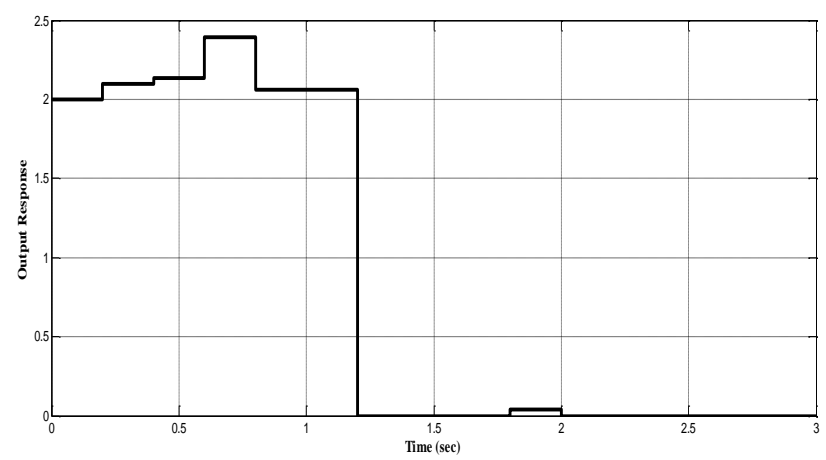

Fig. 9: The control signal

\section{COMPARTION BETWEEN THE CONTROLLER RESULTS}

The comparison between the simulations result are discussed in this section. The controllers MPC and NNMPC are designed with helping of Matlab simulink. Figures 10 and 11 show the step response of the system before adding the controllers and the system with two controllers respectively.

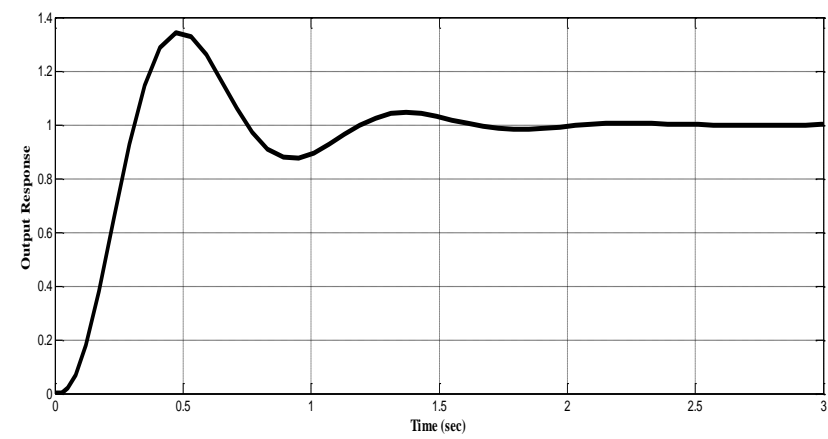

Fig 10: The step response of the system without controller

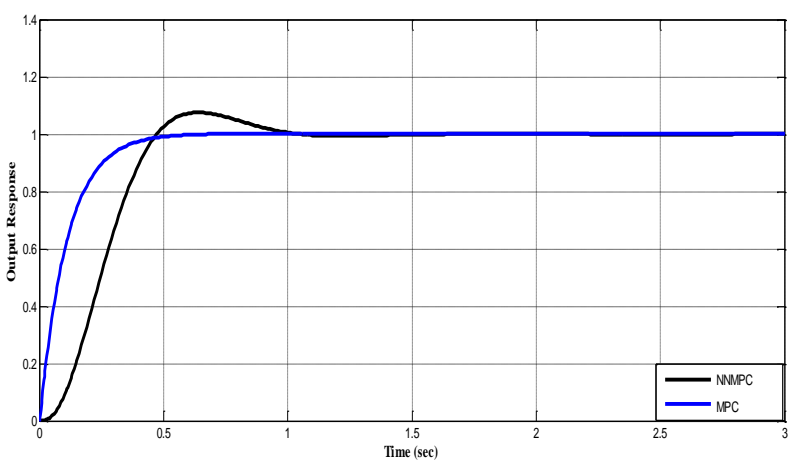

Fig. 11: The comparison between the Controller Results

\section{CONCLUSION}

Most applications, especially medical applications prefer to use the model predictive control because of its high predictive ability in predicting what could be the input values and make the output nearest as possible as to the true value, so it adjusts the input values at every point. The simulation results show that the predictive control gave good results to control the pacemaker but with overshoot. So to remove this problem, a neural network adding for improving the system, which is gives faster setting time with zero overshoot. For the future work, repeat the previous algorithm with different types of controller.

\section{REFERENCES}

[1] Girisha Garg, Asha Rani and Jyoti Yaday, "Intelligent Heart Rate Controller for Cardiac Pacemaker", International Journal of Computer Applications, ISSN: (0975 - 8887), Volume 36- No.7, December 2011.

[2] Achu Govind K. R, Aravind Sekhar R, Vinod B. R, "A Novel Design of Adaptive PID Controller for Cardiac Pacemaker", International Journal of Science and Research (IJSR), ISSN (Online): 2319-7064, Volume 3 Issue 10, October 2014. 
[3] J. W. Shin, J. H. Yoon and. R. Yoon, 2000, "A Study on the Rate-Adaptive Pacemaker by Motion and Respiration using Neuro-Fuzzy", Annual EMBS International Conference, (July 2000).

[4] Neogi, B. ,Ghosh, R., Tarafdar U. and Das A., "Simulation aspect of an artificial pacemaker", Int. Journal. Inf. Tech., pp. 723-727July-Dec. 2010.

[5] Adam Wojtasik, 2000, "Fuzzy logic controller for rateadaptive heart pacemaker", Journal of applied soft computing, pp. 259-270, Aug. 2000.

[6] Xiaowei Wu and Jinggang Zhang, "A Novel Design of PID Controller for Multivariable Control System", IEEE Conference, 2008.
[7] Jyoti Yadav, Asha Rani, Girisha Garg, "Intelligent Heart Rate Controller for Cardiac Pacemaker", International Journal of Computer Applications, 2011.

[8] Steven W. Su., et al., "Nonparametric Hammerstein Model Based Model Predictive Control for Heart Rate Regulation", Conference of the IEEE EMBS, pp. 29842987, 23-26 August, 2007.

[9] Noor S., "Video based Fire Detection using BFO Algorithm with Moving Camera", International Journal of Computer Applications, Vol. 118, No. 16, May 2015.

[10] Kourosh Rahnamai and Kevin Gorman Andrew Gray, "Model Predictive Neural Control of TCP Flow in AQM Network", IEEE, USA, 2006.

[11] The MathWorks http://www.mathworks.com, 2016. 